\title{
FUNCTIONS WHICH OPERATE IN THE FOURIER ALGEBRA OF A COMPACT GROUP
}

\author{
DANIEL RIDER ${ }^{1}$
}

Abstract. Let $A(G)$ be the Fourier algebra of a compact group $G$. It is shown that a function defined on a closed convex subset of the plane operates in $A(G)$ if and only if it is real analytic. This was shown by Helson, Kahane, Katznelson and Rudin when $G$ is locally compact and abelian and by Dunkl when $G$ is compact and contains an infinite abelian subgroup. A direct proof is given of the following lemma which is all that is needed in order to apply the proof of Helson, Kahane, Katznelson and Rudin (\| $\|$ is the Fourier algebra norm).

Lemma. Let $r>0$ and $S_{r}$ be the set of $f \in A(G)$ such that $f$ is real and $\|f\|=r$. Then

$$
\sup _{f \in S_{r}}\left\|e^{i f}\right\|=e^{r} .
$$

1. Introduction. The Fourier algebra, $A(G)$, of a locally compact group $G$ consists of those complex functions which can be written as the convolution, $f * g$, of two functions $f$ and $g$ in $L_{2}(G)$. Eymard [4] has shown that $A(G)$ is a subalgebra of the continuous functions on $G$. When $G$ is abelian and has dual group $\Gamma, A(G)$ is the algebra of Fourier transforms of the group algebra $L_{1}(\Gamma)$. If, in addition, $G$ is compact then $A(G)$ consists of those continuous functions on $G$ having absolutely convergent Fourier series.

If $F$ is a function defined on a subset $E$ of the plane we will say $F$ operates in $A(G)$ provided the composition $F(f)$ belongs to $A(G)$ whenever $f \in A(G)$ and the range of $f$ is contained in $E$.

The functions that operate in $A(G)$ when $G$ is abelian have been completely characterized by Helson, Kahane, Katznelson and Rudin [5]. They show that a function defined on a closed convex set operates if and only if it is real analytic. Recently this has been extended by Dunkl [2] to compact groups which contain infinite abelian subgroups. In this paper we will extend it to all infinite compact groups. Curiously it is not known whether or not an infinite compact group must contain an infinite abelian subgroup so that our result may be a vacuous extension of Dunkl's.

Received by the editors March 2, 1970 and, in revised form, July 30, 1970.

AMS 1970 subject classifications. Primary 43A15, 43A40.

Key words and phrases. Fourier algebra, compact group, functions which operate, real analytic.

1 The author is a fellow of the Alfred P. Sloan Foundation. This research was supported in part by NSF Grant GP-9570.

Copyright (c) 1971, American Mathematical Society 
For a compact group $G$ let $\Gamma$ denote the set of equivalence classes of irreducible unitary representations of $G$. For $\alpha \in \Gamma, T_{\alpha}$ is a representative of the class, $\chi_{\alpha}$ is the character and $d_{\alpha}$ the degree or dimension. The Fourier series of a function $f \in L_{1}(G)$ is given by

$$
f(x) \sim \sum_{\alpha \in \Gamma} d_{\alpha} \operatorname{Tr}\left(A_{\alpha} T_{\alpha}(x)\right) \quad(x \in G)
$$

where $\operatorname{Tr}$ denotes the trace of a matrix and $A_{\alpha}$ is the $d_{\alpha} \times d_{\alpha}$ matrix given by

$$
A_{\alpha}=\int_{G} f\left(x^{-1}\right) T_{\alpha}(x) d x
$$

( $d x$ is the normalized Haar measure of $G$ ).

If $\left|A_{\alpha}\right|$ denotes the absolute value of the matrix $A_{\alpha}$ let $\|f\|$ $=\sum d_{\alpha} \operatorname{Tr}\left(\left|A_{\alpha}\right|\right)$. It is easy to see that $A(G)$ consists of all $f$ with $\|f\|<\infty$. With this norm $A(G)$ is a commutative Banach algebra having $G$ as its maximal ideal space (cf. [3, Chapter 8]). The ArensCalderón-Shilov theorem shows that real analytic functions operate.

Our main contribution is the following lemma.

LEMma 1. Let $r>0$ and $S_{r}$ be the set of $f \in A(G)$ such that $f$ is real and $\|f\|=r$. Then

$$
\sup _{j \in S_{r}}\left\|e^{i j}\right\|=e^{r}
$$

This lemma was shown for abelian groups in [5, Lemma 2.1] and for compact groups with infinite abelian subgroups in [2].

Lemma 1 is all that is needed in order to apply the proof of Helson, Kahane, Katznelson and Rudin [5] (also see 6.6.3 and 6.9.3 of [7]) to obtain

TheOREM 2. Let $G$ be compact. A function defined on a closed convex set operates in $A(G)$ if and only if it is real analytic.

2. The proof of Lemma 1. Henceforth $G$ will be an infinite compact group. Before establishing Lemma 1 we need some facts about $A(G)$.

Proposition 3. If f and $g \in A(G)$ and

$$
\begin{array}{ll}
\int f(y x) \overline{g(x)} d x=0 & (y \in G), \\
\int f(x y) \overline{g(x)} d x=0 & (y \in G),
\end{array}
$$

then $\|f+g\|=\|f\|+\|g\|$. 
Proof. If the Fourier expansions of $f$ and $g$ are given by

$$
f(x) \sim \sum d_{\alpha} \operatorname{Tr}\left(A_{\alpha} T_{\alpha}(x)\right) \text { and } g(x) \sim \sum d_{\alpha} \operatorname{Tr}\left(B_{\alpha} T_{\alpha}(x)\right)
$$

then the integrals in (1) and (2) are given by

$$
\sum d_{\alpha} \operatorname{Tr}\left(B_{\alpha}^{*} A_{\alpha} T_{\alpha}(y)\right)
$$

and

$$
\sum d_{\alpha} \operatorname{Tr}\left(A_{\alpha} B_{\alpha}^{*} T_{\alpha}(y)\right)
$$

where $B_{\alpha}^{*}$ is the adjoint of the matrix $B_{\alpha}$. Since these are zero for all $y$ it follows that

$$
B_{\alpha}^{*} A_{\alpha}=A_{\alpha} B_{\alpha}^{*}=0 .
$$

for all $\alpha \in \Gamma$. It follows from (3) that $\left|A_{\alpha}+B_{\alpha}\right|=\left|A_{\alpha}\right|+\left|B_{\alpha}\right|$. Hence

$$
\begin{aligned}
\|f+g\|= & \sum d_{\alpha} \operatorname{Tr}\left(\left|A_{\alpha}+B_{\alpha}\right|\right)=\sum d_{\alpha} \operatorname{Tr}\left(\left|A_{\alpha}\right|\right) \\
& +\sum d_{\alpha} \operatorname{Tr}\left(\left|B_{\alpha}\right|\right)=\|f\|+\|g\| .
\end{aligned}
$$

If $G$ is infinite and compact it is not known whether $G$ must contain an infinite abelian subgroup. It does not even seem to be known whether $G$ must contain arbitrarily large abelian subgroups. However we can show the following.

Proposition 4. The continuous homomorphic images of $G$ contain arbitrarily large abelian subgroups.

Proof. Clearly we can assume $G$ has an exponent. The finite dimensional representations of $G$ thus have finite images $[1,36.1]$ which are arbitrarily large. Since these images have a common exponent they contain large $p$-groups for some prime $p$ by Sylow's theorem. Since a $p$-group of order $p^{n}$ contains an abelian subgroup of order $p^{a}$ where $a(a+1) \geqq 2 n[6$, Kapital III, Satz 7.3] the proof is complete.

I am indebted to I. M. Isaacs for this proof.

For $\alpha \in \Gamma, T_{\alpha}$ is a homomorphism of $G$ into $U\left(d_{\alpha}\right)$, the group of $d_{\alpha} \times d_{\alpha}$ unitary matrices. Let $\left(T_{\alpha}(x)\right)_{i j}$ denote the matrix entries of $T_{\alpha}(x)$. They will be called entry functions. They depend on the choice of the representative $T_{\alpha}$ but the character $\chi_{\alpha}(x)=\sum_{i}\left(T_{\alpha}(x)\right)_{i i}$ depends only on $\alpha$. Furthermore

$$
\left\|\left(T_{\alpha}\right)_{i j}\right\|=1 \text { and }\left(T_{\alpha}(e)\right)_{i j}=\delta_{i j}
$$

where $e$ is the identity element of $G$.

Let $B$ be a closed subgroup of $G$ and $K$ a closed normal subgroup such that $B / K$ is abelian. If $T_{\alpha}$ is an irreducible representation such 
that $K \subset$ Kernel $T_{\alpha}$ then $T_{\alpha}(B)$ is abelian so that an equivalent representation, also called $T_{\alpha}$, can be chosen with the property that the matrices $T_{\alpha}(a)$ are diagonal for all $a \in B$. The diagonal entry functions for this $T_{\alpha}$ are thus one-dimensional representations (or characters) of $B$. They also can be considered as characters of $B / K$. Furthermore every (one-dimensional) character of $B / K$ arises in this way. If $\phi$ is such an entry function we also have

$$
\phi(a g)=\phi(g a)=\phi(a) \phi(g) \quad(a \in B, g \in G) .
$$

In what follows we use the notation

$$
\phi^{-1}(x)=\overline{\phi(x)} \text {. }
$$

If $\phi_{1}, \phi_{2}, \cdots, \phi_{t}$ are functions then for $1 \leqq i \leqq t$ let

$$
\theta_{i}(x)=\sum \phi_{s_{1}}^{ \pm 1}(x) \phi_{s_{2}}^{ \pm 1}(x) \cdots \phi_{s_{i}}^{ \pm 1}(x),
$$

the sum being extended over all $i$-tuples $1 \leqq s_{1}<s_{2}<\cdots<s_{i} \leqq t$ and all sequences of $\pm 1 . \theta_{0}(x)=1$.

Proposition 5. Given an integer $t$ there exist $t$ diagonal entry functions $\phi_{1}, \cdots, \phi_{t}$ such that for all complex numbers $z_{0}, z_{1}, \cdots, z_{t}$

$$
\left\|\sum_{0}^{t} z_{i} \theta_{i}\right\|=\sum_{0}^{t}\left|z_{i}\right|\left\|\theta_{i}\right\| .
$$

Proof. There is an integer $N$ such that any locally compact abelian group with order $\geqq N$ has $t$ characters $\phi_{1}, \cdots, \phi_{t}$ such that if $s_{1}<s_{2}<\cdots<s_{i}, r_{1}<r_{2}<\cdots<r_{j}$ and

$$
\phi_{s_{1}}^{ \pm 1} \phi_{s_{2}}^{ \pm 1} \cdots \phi_{s_{i}}^{ \pm 1} \phi_{r_{1}}^{ \pm 1} \cdots \phi_{r_{j}}^{ \pm 1}=1
$$

for some choice of \pm 1 then $i=j$.

By Proposition 4 there is an abelian group, $B / K$, of order $\geqq N$ in some homomorphic image of $G$. By the remarks above the $t$ characters on $B / K$ which satisfy ( 7$)$ can be extended to diagonal entry functions on $G$. I claim that if $i \neq j, s_{1}<\cdots<s_{i}$ and $r_{1}<\cdots<r_{j}$ then, for all choices of \pm 1 ,

$$
I=\int_{G} \phi_{s_{1}}^{ \pm 1}(y x) \phi_{s_{2}}^{ \pm 1}(y x) \cdots \phi_{s_{i}}^{ \pm 1}(y x) \phi_{r_{i}}^{ \pm 1}(x) \cdots \phi_{r_{j}}^{ \pm 1}(x) d x=0
$$

for all $y \in G$. For if $x$ is replaced by $x a$ (with $a \in B$ ) in the integral of (8) then (5) and the invariance of $d x$ show that

$$
I=I \phi_{s_{1}}^{ \pm 1}(a) \cdots \phi_{s_{i}}^{ \pm 1}(a) \phi_{r_{1}}^{ \pm 1}(a) \cdots \phi_{r_{j}}^{ \pm 1}(a) .
$$


By (7) this is possible only if $I=0$.

From (8) it follows at once that

$$
\int_{G} \theta_{i}(y x) \overline{\theta_{j}(x)} d x=\int_{G} \theta_{i}(y x) \theta_{j}(x) d x=0 \quad(i \neq j ; y \in G) .
$$

Similarly

$$
\int_{G} \theta_{i}(x y) \overline{\theta_{j}(x)} d x=0 \quad(i \neq j ; y \in G) .
$$

These last equations allow us to apply Proposition 3 inductively to the $\theta_{i}$ in order to obtain (6).

Proof of Lemma 1. Fix $r>0$. Let $t$ be an integer, let $\phi_{1}, \phi_{2}, \cdots, \phi_{t}$ be as in Proposition 5 and define

$$
f(x)=\frac{r}{2 t} \sum_{i=1}^{t} \phi_{i}(x)+\overline{\phi_{i}(x)}
$$

$f$ is real and

$$
\|f\|=r .
$$

Now if $1 \leqq n \leqq t$ then

$$
f^{n}(x)=\left(\frac{r}{2 t}\right)^{n} n ! \theta_{n}(x)+B_{n}(x)
$$

where

$$
B_{n}(x)=\left(\frac{r}{2 t}\right)^{n} \sum \phi_{s_{1}}^{ \pm 1}(x) \cdots \phi_{s_{n}}^{ \pm 1}(x) ;
$$

this sum is over all $n$-tuples $\left(s_{1}, \cdots, s_{n}\right)$ such that at least two of the $s_{i}$ are the same and is over all choices of \pm 1 .

Now since $\left\|\phi_{i}\right\|=1$ and the sum in (12) has $(2 t)^{n}-2^{n} n !\left(\begin{array}{l}b \\ n\end{array}\right)$ elements, it follows that

$$
\left\|B_{n}\right\| \leqq r^{n}\left[1-\frac{t !}{(t-n) ! t^{n}}\right] .
$$

On the other hand, the sum which gives $\theta_{n}$ has $2^{n}\left(\begin{array}{l}l \\ n\end{array}\right)$ elements so that

$$
\left\|\theta_{n}\right\| \geqq \theta_{n}(e)=2^{n}\left(\begin{array}{l}
t \\
n
\end{array}\right) .
$$


It then follows from (11), (6), (14), (10) and (13) that

$$
\begin{aligned}
\left\|e^{i f}\right\| & \geqq\left\|\sum_{0}^{\infty} \frac{(i f)^{n}}{n !}\right\| \\
& \geqq\left\|\sum_{0}^{t}\left(\frac{i r}{2 t}\right)^{n} \theta_{n}\right\|-\sum_{0}^{t} \frac{\left\|B_{n}\right\|}{n !}-\sum_{t+1}^{\infty} \frac{\left\|f^{n}\right\|}{n !} \\
& \geqq \sum_{0}^{t}\left(\frac{r}{2 t}\right)^{n} 2^{n}\left(\begin{array}{l}
t \\
n
\end{array}\right)-\sum_{0}^{t} \frac{r^{n}}{n !}\left[1-\frac{t !}{(t-n) ! t^{n}}\right]-\sum_{t+1}^{\infty} \frac{r^{n}}{n !} \\
& \geqq 2 \sum_{0}^{t}\left(\frac{r}{t}\right)^{n}\left(\begin{array}{l}
t \\
n
\end{array}\right)-e^{r} .
\end{aligned}
$$

Thus, letting $S_{r}=\{f: f$ is real; $\|f\|=r\}$, we obtain

$$
\begin{gathered}
\sup _{f \in S_{r}}\left\|e^{i f}\right\| \geqq \sup _{t} 2 \sum_{0}^{t}\left(\frac{r}{t}\right)^{n}\left(\begin{array}{l}
t \\
n
\end{array}\right)-e^{r}=e^{r} . \\
\text { REFERENCES }
\end{gathered}
$$

\section{REFERENCES}

1. C. W. Curtis and I. Reiner, Representation theory of finite groups and associative algebras, Pure and Appl. Math., vol. XI, Interscience, New York, 1962. MR 26 \#2519.

2. C. F. Dunkl, Functions that operate in the Fourier algebra of a compact group, Proc. Amer. Math. Soc. 21 (1969), 540-544. MR 39 \#717.

3. C. Dunkl and D. Ramirez, Topics in harmonic analysis, Appleton-CenturyCrofts, New York, 1971.

4. P. Eymard, L'algèbre de Fourier d'un groupe localement compact, Bull. Soc. Math. France 92 (1964), 181-236. MR 37 \#4208.

5. H. Helson, J.-P. Kahane, Y. Katznelson and W. Rudin, The functions which operate on Fourier transforms, Acta Math. 102 (1959), 135-157. MR 22 \#6980.

6. B. Huppert, Endliche Gruppen. I, Die Grundlehren der math. Wissenschaften, Band 134, Springer-Verlag, Berlin and New York, 1967. MR 37 \#302.

7. W. Rudin, Fourier analysis on groups, Interscience Tracts in Pure and Appl. Math., no. 12, Interscience, New York, 1962. MR 27 \#2808.

University of Wisconsin, Madison, Wisconsin 53706 